\title{
Renormalization Group Equation and QCD Coupling Constant in the Presence of SU(3) Chromo-Electric Field
}

\author{
Gouranga C. Nayak, ${ }^{1, *}$ \\ ${ }^{1}$ Department of Physics, University of Arizona, Tucson, AZ 85721, USA
}

(Dated: October 28, 2018)

\begin{abstract}
We solve renormalization group equation in QCD in the presence of SU(3) constant chromoelectric field $E^{a}$ with arbitrary color index $a=1,2, \ldots 8$ and find that the QCD coupling constant $\alpha_{s}$ depends on two independent casimir/gauge invariants $C_{1}=\left[E^{a} E^{a}\right]$ and $C_{2}=\left[d_{a b c} E^{a} E^{b} E^{c}\right]^{2}$ instead of one gauge invariant $C_{1}=\left[E^{a} E^{a}\right]$. The $\beta$ function is derived from the one-loop effective action. This coupling constant may be useful to study hadron formation from color flux tubes/strings at high energy colliders and to study quark-gluon plasma formation at RHIC and LHC.

PACS numbers: PACS: 11.15.-q, 11.15.Me, 12.38.Cy, 11.15.Tk
\end{abstract}

*Electronic address: nayak@physics.arizona.edu 


\section{INTRODUCTION}

Although quantum chromodynamics describes the interaction among quarks and gluons, the classical color field is used in many experimental situations, especially to model the non-perturbative physics. Quark and gluon production from strong chromo-electric field via Schwinger mechanism [1, 2, 3, 4] itself is a non-perturbative effect. This mechanism is often used in PYTHIA generator [5] to study low $p_{T}$ hadron production at collider experiments. At RHIC and LHC heavy-ion colliders the classical color field play an important role to study production of quark-gluon plasma [6]. Color glass condensate provide a natural framework to determine the initial condition on the classical color field at RHIC and LHC [7].

In these situations it is necessary to know how QCD coupling constant depends on $\mathrm{SU}(3)$ color field. In this paper we solve the renormalization group equation in QCD in the presence of $\mathrm{SU}(3)$ constant chromo-electric field $E^{a}$ with arbitrary color index $a=1,2, \ldots 8$. Using background field method in QCD we derive $\beta$ function from the one loop effective action of quark and gluon in the presence of constant chromo-electric field $E^{a}$. Using these two facts we determine the exact dependence of the QCD coupling constant $\alpha_{s}$ on chromo-electric field $E^{a}$ in $\mathrm{SU}(3)$.

The paper is organized as follows. In section II we solve renormalization group equation in QCD in the presence of $\mathrm{SU}(3)$ chromo-electric field. In section III we derive $\beta$ function from one-loop effective action. In section IV we discuss the dependence of QCD coupling constant on the second casimir invariant in $\mathrm{SU}(3)$. We present our conclusions in section $\mathrm{V}$.

\section{RENORMALIZATION GROUP EQUATION IN QCD IN SU(3) CHROMO- ELECTRIC FIELD}

In the background field method of QCD [8, 9] the total gauge field is the sum of classical background field $A_{\mu}^{a}$ and quantum gluon field $Q_{\mu}^{a}$. The gauge fixing term depends on the

background field $A_{\mu}^{a}$. As pointed out in [9] it is not necessary to renormalize the quantum gluon fields $Q_{\mu}^{a}$ and the ghost fields. Gauge fixing parameter renormalization is also not necessary because the physical result is gauge fixing parameter independent. Hence the background field $A_{\mu}^{a}$ and coupling constant $g$ need to be renormalized. We define the bare 
quantities in terms of the renormalized one as follows [9]

$$
A_{\mu}^{a}=Z_{A}^{-\frac{1}{2}} A_{\mu r}^{a}, \quad g=Z_{g} g_{r}
$$

This gives

$$
F_{\mu \nu}^{a}[A]=Z_{A}^{-\frac{1}{2}}\left[\partial_{\mu} A_{\nu r}^{a}-\partial_{\nu} A_{\mu r}^{a}+Z_{A}^{-\frac{1}{2}} Z_{g} g f^{a b c} A_{\mu r}^{b} A_{\nu r}^{c}\right]
$$

Since $F_{\mu \nu}^{a}[A]$ transforms covariantly with respect to gauge transformation we find from the above equation

$$
Z_{g}=Z_{A}^{\frac{1}{2}}, \quad \text { which gives, } \quad \beta=-g \gamma,
$$

where we have used

$$
\beta=-g \mu \frac{\partial}{\partial \mu} \operatorname{Ln} Z_{g}, \quad \text { and } \quad \gamma=\frac{1}{2} \mu \frac{\partial}{\partial \mu} \operatorname{Ln} Z_{A} .
$$

The renormalization group equation for the effective action $\Gamma$ can be written as [10]

$$
\left[\mu^{2} \frac{\partial}{\partial \mu^{2}}+\beta \frac{\partial}{\partial g}+\gamma \int d^{4} x A_{\mu}^{a} \frac{\delta}{\delta A_{\mu}^{a}}\right] \Gamma=0 .
$$

The effective action may be written in terms of the 1PI Green's function via

$$
\Gamma=\sum_{n} \frac{1}{n !} \int d^{4} x_{1} \ldots . . d^{4} x_{n} \Gamma_{\mu_{1}, \ldots . \mu_{n}}^{(n)}\left(x_{1}, \ldots x_{n}\right) A_{\mu_{1}}^{a_{1}, \ldots . . a_{n}} \ldots A_{\mu_{n}}^{a_{n}} .
$$

Using eq. (6) in (5) we find

$$
\left[\mu^{2} \frac{\partial}{\partial \mu^{2}}+\beta \frac{\partial}{\partial g}+n \gamma\right] \Gamma^{(n)}=0
$$

which is the familiar form of the renormalization group equation in QCD [11].

There is another way to expand the effective action [2, 10]. Instead of expanding in powers of $A_{\mu}^{a}$ one can expand in powers of momentum. In coordinate space it has the form

$$
\Gamma=\int d^{4} x \mathcal{L}=\int d^{4} x\left[-V(A)+\frac{1}{2}\left(\partial_{\mu} A_{\nu}^{a}\right)^{2} Z_{A}+\ldots . .\right.
$$

Hence we will use eq. (5) instead of (7) for the differential equation of the renormalization group. In the presence of constant chromo-electric field the one loop effective action for gluon

$$
\mathcal{L}^{(1)}=\sum_{j=1}^{3} \mathcal{L}_{j}^{(1)}
$$


depends on three independent gauge and Lorentz invariant eigenvalues $\lambda_{j}$ of $f^{a b c} E^{c}$ in $\mathrm{SU}(3)$

$$
\lambda_{1}^{2}=\frac{C_{1}}{2}[1-\cos \theta], \quad \lambda_{2,3}^{2}=\frac{C_{1}}{2}\left[1+\cos \left(\frac{\pi}{3} \pm \theta\right)\right], \quad \cos 3 \theta=-1+\frac{6 C_{2}}{C_{1}^{3}} .
$$

Hence the renormalized effective lagrangian density $\mathcal{L}_{j}$ depends on $\lambda_{j}$. In terms of gauge and Lorentz invariant variables, the renormalization group equation becomes

$$
\left[\mu^{2} \frac{\partial}{\partial \mu^{2}}+\beta \frac{\partial}{\partial g}+\gamma \lambda_{j} \frac{\partial}{\partial \lambda_{j}}\right] \mathcal{L}_{j}=0
$$

In order to solve this differential equation we define dimensionless Lagrangian density

$$
\overline{\mathcal{L}}_{j}=\frac{\mathcal{L}_{j}}{\frac{1}{3} \lambda_{j}^{2}}
$$

which can only depend on the dimensionless quantity

$$
t_{j}=\ln r_{j}=\ln \frac{g \lambda_{j}}{\mu^{2}} .
$$

We find from eq. (11)

$$
\frac{1}{\overline{\mathcal{L}}_{j}\left(t_{j}\right)} \frac{\partial \overline{\mathcal{L}}_{j}\left(t_{j}\right)}{\partial t_{j}}+\frac{\bar{\beta}}{\overline{\mathcal{L}}_{j}\left(t_{j}\right)} \frac{\partial \overline{\mathcal{L}}_{j}\left(t_{j}, g\right)}{\partial g}=-2 \bar{\gamma}
$$

where

$$
\bar{\beta}=\frac{\beta}{(\gamma-1)}, \quad \text { and } \quad \bar{\gamma}=\frac{\gamma}{(\gamma-1)} .
$$

Solving the differential eq. (14) we find:

$$
-\overline{\mathcal{L}}_{j}\left(t_{j}\right) d t_{j}=-\frac{\overline{\mathcal{L}}_{j}\left(t_{j}\right)}{\bar{\beta}} d g=\frac{d \overline{\mathcal{L}}_{j}\left(t_{j}\right)}{2 \bar{\gamma}}
$$

which gives

$$
\frac{d g}{d t_{j}}=\bar{\beta}
$$

In this paper we consider $\alpha_{s}$ at the one loop level and take the $\beta$ function of the form

$$
\bar{\beta}(g)=-\bar{\beta}_{0} g^{3} .
$$

We find from eq. (17) the QCD coupling constant

$$
\alpha_{s}\left(\lambda_{j}\right)=\frac{g^{2}\left(t_{j}\right)}{4 \pi}=\frac{g^{2}}{4 \pi\left[1+2 \bar{\beta}_{0} g^{2} t_{j}\right]}=\frac{\alpha_{s}}{\left[1+4 \pi \bar{\beta}_{0} \alpha_{s} \log \left(\frac{g^{2} \lambda_{j}^{2}}{\mu^{4}}\right)\right]}=\frac{1}{4 \pi \bar{\beta}_{0} \log \left(\frac{g^{2} \lambda_{j}^{2}}{\Lambda^{4}}\right)}
$$


where

$$
\Lambda=\mu e^{\left(-1 /\left(4 \bar{\beta}_{0} g^{2}\right)\right)}, \quad \quad \alpha_{s}=\frac{g^{2}}{4 \pi}
$$

For the quark case the $\beta$ functions are different and the eigenvalues are in fundamental representation of $\mathrm{SU}(3)$. Three independent gauge and Lorentz invariant eigenvalues of $T_{i j}^{a} E^{a}$ for the quark case are given by

$$
\lambda_{1}=\sqrt{\frac{C_{1}}{3}} \cos \theta, \quad \lambda_{2,3}=\sqrt{\frac{C_{1}}{3}} \cos \left(\frac{2 \pi}{3} \pm \theta\right), \quad \cos ^{2} 3 \theta=\frac{3 C_{2}}{C_{1}^{3}} .
$$

All now remains is to find the $\beta$ functions from one-loop effective action which we will derive in the next section.

\section{III. $\beta$ FUNCTION IN QCD FROM ONE-LOOP EFFECTIVE ACTION}

The one-loop effective lagrangian density for gluon in the presence of $\mathrm{SU}(3)$ constant chromo-electric field $E^{a}$ with $a=1,2, \ldots 8$ is given by [1]

$$
\mathcal{L}_{j}^{(1)}=\frac{1}{8 \pi^{2}} \int_{0}^{\infty} \frac{d s}{s^{2}}\left[g \lambda_{j} \frac{\cos 2 g \lambda_{j} s}{\sin g \lambda_{j} s}-\frac{1}{s}\right] .
$$

Expanding sin and cos functions we get

$$
\mathcal{L}_{j}^{(1)}=\frac{1}{8 \pi^{2}} \int_{0}^{\infty} \frac{d s}{s^{2}}\left[-\frac{11 g^{2} \lambda_{j}^{2} s}{6}+\frac{39 g^{4} \lambda_{j}^{4}}{240} s^{3}+\ldots\right] .
$$

Since $s$ has dimension of length, the ultra violate divergence occurs at $s \rightarrow 0$ which leads to charge renormalization [2]. The ultra violate divergent term in eq. (23) is $\frac{1}{8 \pi^{2}} \int_{0}^{\infty} \frac{d s}{s} \frac{11 g^{2} \lambda_{j}^{2}}{6}$. We write eq. (22) as follows

$$
\mathcal{L}_{j}^{(1)}=\frac{1}{8 \pi^{2}} \int_{0}^{\infty} \frac{d s}{s^{2}}\left[g \lambda_{j} \frac{\cos 2 g \lambda_{j} s}{\sin g \lambda_{j} s}+\frac{11 g^{2} \lambda_{j}^{2} s}{6}-\frac{1}{s}\right]-\frac{1}{8 \pi^{2}} \int_{0}^{\infty} \frac{d s}{s} \frac{11 g^{2} \lambda_{j}^{2}}{6} .
$$

The $s$ integration involving the square bracket term is now finite. We can obtain the $\beta$ function from the coefficient of the divergent term by renormalization procedure by adding the counter term $Z \mathcal{L}_{0}$. We put a cut-off for the infrared limit at $s=s_{0}$ and by change to the dimensionless variable $s_{j} \rightarrow s g \lambda_{j}$. We find

$$
\mathcal{L}_{j}^{(1)}=\frac{g^{2} \lambda_{j}^{2}}{8 \pi^{2}} \int_{0}^{\infty} \frac{d s_{j}}{s_{j}^{2}}\left[\frac{\cos 2 s_{j}}{\sin s_{j}}+\frac{11}{6}-\frac{1}{s_{j}}\right]-\frac{1}{8 \pi^{2}} \int_{0}^{g \lambda_{j} s_{0}} \frac{d s_{j}}{s_{j}} \frac{11 g^{2} \lambda_{j}^{2}}{6} .
$$


The free lagrangian density is given by

$$
\mathcal{L}_{0}=\frac{E^{a} E^{a}}{2}=\frac{1}{3} \sum_{j=1}^{3} \lambda_{j}^{2}=\sum_{j=1}^{3} \mathcal{L}_{0}^{j} .
$$

Adding the counter term $\left(Z \mathcal{L}_{0}^{j}\right)$ to eq. (25) the renormalized lagrangian density becomes

$$
\mathcal{L}_{j}\left(t_{j}\right)=Z \mathcal{L}_{0}^{j}+\mathcal{L}_{j}^{(1)}
$$

The renormalization condition is then given by

$$
\left.\frac{\mathcal{L}_{j}\left(t_{j}\right)}{\mathcal{L}_{0}^{j}}\right|_{t_{j}=0}=1
$$

We find from the above equation

$$
Z=1-3 \frac{g^{2}}{8 \pi^{2}} \int_{0}^{\infty} \frac{d s_{j}}{s_{j}^{2}}\left[\frac{\cos 2 s_{j}}{\sin s_{j}}+\frac{11}{6}-\frac{1}{s_{j}}\right]+\frac{3}{8 \pi^{2}} \int_{0}^{g \mu^{2} s_{0}} \frac{d s_{j}}{s_{j}} \frac{11 g^{2}}{6}
$$

which when used in eq. (27) gives the renormalized Lagrangian density

$$
\overline{\mathcal{L}}_{j}=\frac{\mathcal{L}_{j}\left(t_{j}\right)}{\mathcal{L}_{0}^{j}}=1-\frac{11}{16 \pi^{2}} g^{2} t_{j}
$$

The $\beta$ function can be obtained from the renormalized Lagrangian density. From eqs. (14), (12) and (28) we find

$$
\bar{\gamma}=-\left.\frac{1}{2} \frac{\partial \overline{\mathcal{L}}_{j}\left(t_{j}\right)}{\partial t_{j}}\right|_{t_{j}=0}
$$

Using eqs. (15) and (3) in the above equation we find

$$
\bar{\beta}=\left.\frac{g}{2} \frac{\partial \overline{\mathcal{L}}_{j}\left(t_{j}\right)}{\partial t_{j}}\right|_{t_{j}=0}
$$

Using eq. (32) we find from eq. (30)

$$
\bar{\beta}=-\frac{11}{32 \pi^{2}} g^{3}
$$

which gives the $\beta$ function for a gluon loop

$$
\bar{\beta}_{0}{ }^{g}=\frac{11}{32 \pi^{2}}
$$

where we have used eq. (18).

The effective Lagrangian density for massless quark is given by [1]

$$
\mathcal{L}_{j}^{(1)}=-\frac{1}{8 \pi^{2}} \int_{0}^{\infty} \frac{d s}{s^{2}}\left[g \lambda_{j} \frac{\cos g \lambda_{j} s}{\sin g \lambda_{j} s}-\frac{1}{s}\right] .
$$


Expanding sin and cos functions we get

$$
\mathcal{L}^{(1)}=-\frac{1}{8 \pi^{2}} \int_{0}^{\infty} \frac{d s}{s}\left[\frac{g^{2} \lambda_{j}^{2}}{3}+\frac{g^{4} \lambda_{j}^{4}}{20} s^{2}+\ldots\right] .
$$

The coefficient of the ultra violate divergent term (as $s \rightarrow 0)$ is $\frac{1}{3}$. The free Lagrangian density is given by

$$
\mathcal{L}_{0}=\frac{E^{a} E^{a}}{2}=\sum_{j=1}^{3} \lambda_{j}^{2}=\sum_{j=1}^{3} \mathcal{L}_{0}^{j} .
$$

Carrying out similar renormalization analysis as for gluons we obtain the renormalized Lagrangian density

$$
\overline{\mathcal{L}}_{j}=1+\frac{1}{24 \pi^{2}} g^{2} t_{j}
$$

which gives the $\beta$ function for a quark loop

$$
\bar{\beta}_{0}{ }^{q}=-\frac{1}{48 \pi^{2}} .
$$

To summarize, eq. (19) describes evolution of QCD coupling constant in the presence of $\mathrm{SU}(3)$ constant chromo-electric field, together with eqs. (34) and (39) as $\bar{\beta}_{0}$ functions for a gluon and quark loop respectively.

\section{QCD COUPLING CONSTANT AND SECOND CASIMIR INVARIANT IN $\mathrm{SU}(3)$}

It can be seen that the two independent casimir invariants $C_{1}=\left[E^{a} E^{a}\right]$ and $C_{2}=$ $\left[d_{a b c} E^{a} E^{b} E^{c}\right]^{2}$ in $\mathrm{SU}(3)$ are gauge invariant with respect to the gauge transformation

$$
\left(T^{a} E^{a}\right)^{\prime}=U\left(T^{a} U^{a}\right) U^{-1}, \quad U=e^{i T^{a} \omega^{a}(x)} .
$$

Let us denote the vector $\vec{E}$ in 8-dimensional color space in $\mathrm{SU}(3)$ with components $E_{a}=$ $\left(E_{1}, E_{2}, \ldots . ., E_{8}\right)$. It can be seen that while the first casimir invariant $C_{1}$ is independent of the direction of the vector $\vec{E}$, the second casimir invariant $C_{2}$ may depend on the direction of $\vec{E}$ in 8-dimensional color space even if it is gauge invariant. This is because of presence of $d_{a b c}$ whose components are not equal to each other [12]. These two independent casimir invariants satisfy the limit

$$
0 \leq \frac{3 C_{2}}{C_{1}^{3}} \leq 1
$$


Eq. (41) gives the limit

$$
0 \leq \theta \leq \frac{2 \pi}{3}
$$

when the $\lambda_{j}$ 's are given by eq. (10) in the adjoint representation of $\mathrm{SU}(3)$ and

$$
\frac{\pi}{6} \leq \theta \leq \frac{\pi}{2}
$$

when the $\lambda_{j}$ 's are given by eq. (21) in the fundamental representation of $\mathrm{SU}(3)$.

Since $\theta$ appears inside $\log$ in $\alpha_{s}\left(\lambda_{j}\right)$ it is useful to check that this $\theta$ dependence is under control for this one loop calculation. When the $\lambda_{j}$ 's are given by eq. (10), it can be checked that the one-loop result of $\alpha_{s}\left(\lambda_{j}\right)$ in the maximum allowed range $0 \leq \theta \leq \frac{2 \pi}{3}$ (see eq. (42)) is under control only for asymptotically very large value of $C_{1}=\left[E^{a} E^{a}\right]$ (see below). Similarly, when the $\lambda_{j}$ 's are given by eq. (21), the one-loop result of $\alpha_{s}\left(\lambda_{j}\right)$ in the maximum allowed range $\frac{\pi}{6} \leq \theta \leq \frac{\pi}{2}$ (see eq. (43) ) is under control only for asymptotically very large value of $C_{1}=\left[E^{a} E^{a}\right]$.

For realistic values of $C_{1}=\left[E^{a} E^{a}\right], g$ and $\Lambda$ at RHIC and LHC we can determine the range of $\theta$ for which $0<\alpha_{s} \leq 1$ in this one-loop calculation of $\alpha_{s}$. For example, by using eq. (10) we find from $0<\alpha_{s} \leq 1$, the range

$$
\cos ^{-1}\left[1-\frac{2 \Lambda^{4}}{g^{2} C_{1}} e^{\frac{1}{4 \pi \beta_{0}}}\right] \leq \theta \leq-\pi / 3+\cos ^{-1}\left[\frac{2 \Lambda^{4}}{g^{2} C_{1}} e^{\frac{1}{4 \pi \beta_{0}}}-1\right] .
$$

It can be seen that when $C_{1}=\left[E^{a} E^{a}\right]$ is asymptotically very large, say, $C_{1} \rightarrow \infty$ we find from eq. (44), $0 \leq \theta \leq \frac{2 \pi}{3}$ which reproduces the maximum allowed range as given by eq. (42). For realistic values of $C_{1}=\left[E^{a} E^{a}\right], g$ and $\Lambda$ at RHIC and LHC the range in eq. (44) may be be very close to the maximum allowed range in eq. (42). For example, if we choose $\Lambda=0.2 \mathrm{GeV}, \mathrm{g}=3$ and $C_{1}=E^{a} E^{a}=1000 \mathrm{GeV}^{4}$ (which may be a reasonable value at LHC) we find from eq. (44), $0.0021 \leq \theta \leq 2.092$. This range of $\theta$ is very close to the maximum allowed range $0 \leq \theta \leq \frac{2 \pi}{3}$ or $0 \leq \theta \leq 2.094$ as given by eq. (42).

Similarly, by using eq. (21) we find from $0<\alpha_{s} \leq 1$, the range

$$
\frac{2 \pi}{3}-\cos ^{-1}\left[\sqrt{\frac{3 \Lambda^{4}}{g^{2} C_{1}} e^{\frac{1}{4 \pi \beta_{0}}}}\right] \leq \theta \leq \cos ^{-1}\left[\sqrt{\frac{3 \Lambda^{4}}{g^{2} C_{1}} e^{\frac{1}{4 \pi \beta_{0}}}}\right] .
$$

It can be seen that when $C_{1}=\left[E^{a} E^{a}\right]$ is asymptotically very large, say, $C_{1} \rightarrow \infty$ we find from eq. (45), $\frac{\pi}{6} \leq \theta \leq \frac{\pi}{2}$ which reproduces the maximum allowed range as given by eq. (43). If we choose the previous values, $\Lambda=0.2 \mathrm{GeV}, \mathrm{g}=3$ and $C_{1}=E^{a} E^{a}=1000 \mathrm{GeV}^{4}$ we 
find from eq. (45), $0.526 \leq \theta \leq 1.569$. This range of $\theta$ is very close to the maximum allowed range $\frac{\pi}{6} \leq \theta \leq \frac{\pi}{2}$ or $0.5236 \leq \theta \leq 1.571$ as given by eq. (43).

In Fig. 1 we present the result of $\alpha_{s}\left(\lambda_{j}\right)$ as function of $\theta$ for fixed values of $C_{1}=\left[E_{a} E_{a}\right]$. We have used $g=3$ and $\Lambda=200 \mathrm{MeV}$. The scale $\lambda_{j}$ 's are given by eq. (10). The range of $\theta$ in Fig. 1 is given by eq. (44). The upper, middle and lower solid lines are the results of $\alpha_{s}\left(\lambda_{1}\right)$ for $C_{1}=10,1000$ and $100000 \mathrm{GeV}^{4}$ respectively. The upper, middle and lower dotted lines are the results of $\alpha_{s}\left(\lambda_{2}\right)$ for $C_{1}=10,1000$ and $100000 \mathrm{GeV}^{4}$ respectively. The upper, middle and lower dashed lines are the results of $\alpha_{s}\left(\lambda_{3}\right)$ for $C_{1}=10,1000$ and 100000 $\mathrm{GeV}^{4}$ respectively. It can be seen from Fig. 1 that the $\theta$ dependence is under control for the entire range of $\theta$ as given by eq. (44) which is very close to the actual maximum range $0 \leq \theta \leq \frac{2 \pi}{3}$ in eq. (42).

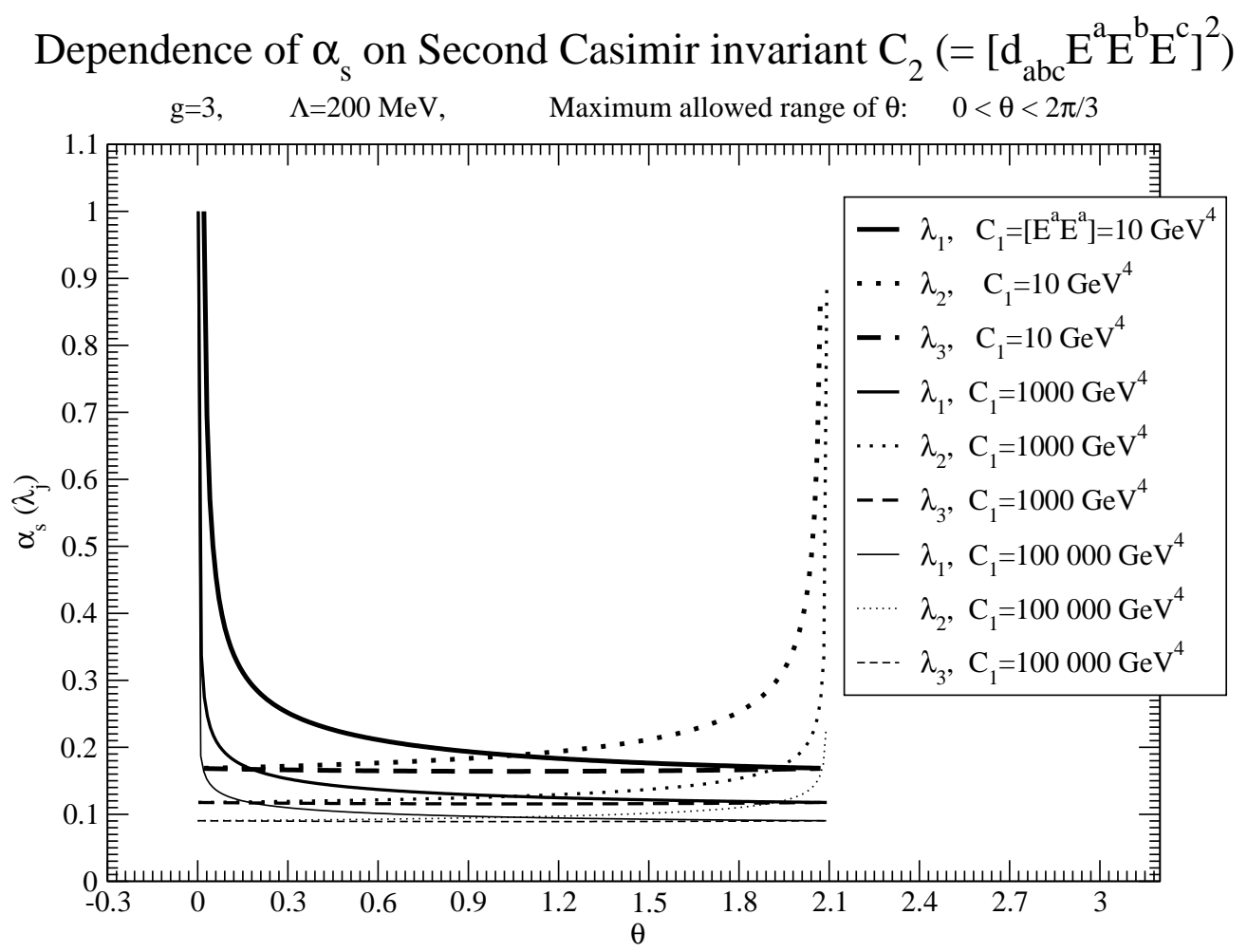

FIG. 1: QCD coupling constant in the presence of SU(3) chromo-electric field as function $\theta$ for fixed values of first casimir invariant $C_{1}=E^{a} E^{a}$. The $\lambda_{j}$ 's used are from eq. (10). Remember that $0 \leq \theta \leq \frac{2 \pi}{3}$ is the maximum range when the $\lambda_{j}$ 's are given by eq. (10).

In Fig. 2 we present the result of $\alpha_{s}\left(\lambda_{j}\right)$ as function of $\theta$ for fixed values of $C_{1}=\left[E_{a} E_{a}\right]$. We have used $g=3$ and $\Lambda=200 \mathrm{MeV}$. The scale $\lambda_{j}$ 's are given by eq. (21). The range of 
$\theta$ in Fig. 2 is given by eq. (45). The upper, middle and lower solid lines are the results of $\alpha_{s}\left(\lambda_{1}\right)$ for $C_{1}=10,1000$ and $100000 \mathrm{GeV}^{4}$ respectively. The upper, middle and lower dotted lines are the results of $\alpha_{s}\left(\lambda_{2}\right)$ for $C_{1}=10,1000$ and $100000 \mathrm{GeV}^{4}$ respectively. The upper, middle and lower dashed lines are the results of $\alpha_{s}\left(\lambda_{3}\right)$ for $C_{1}=10,1000$ and 100000 $\mathrm{GeV}^{4}$ respectively. It can be seen from Fig. 2 that the $\theta$ dependence is under control for the entire range of $\theta$ as given by eq. (45) which is very close to the actual maximum range $\frac{\pi}{6} \leq \theta \leq \frac{\pi}{2}$ in eq. (43).

\section{Dependence of $\alpha_{s}$ on Second Casimir invariant $C_{2}\left(=\left[d_{a b c} E^{a} E^{b} E^{c}\right]^{2}\right)$}

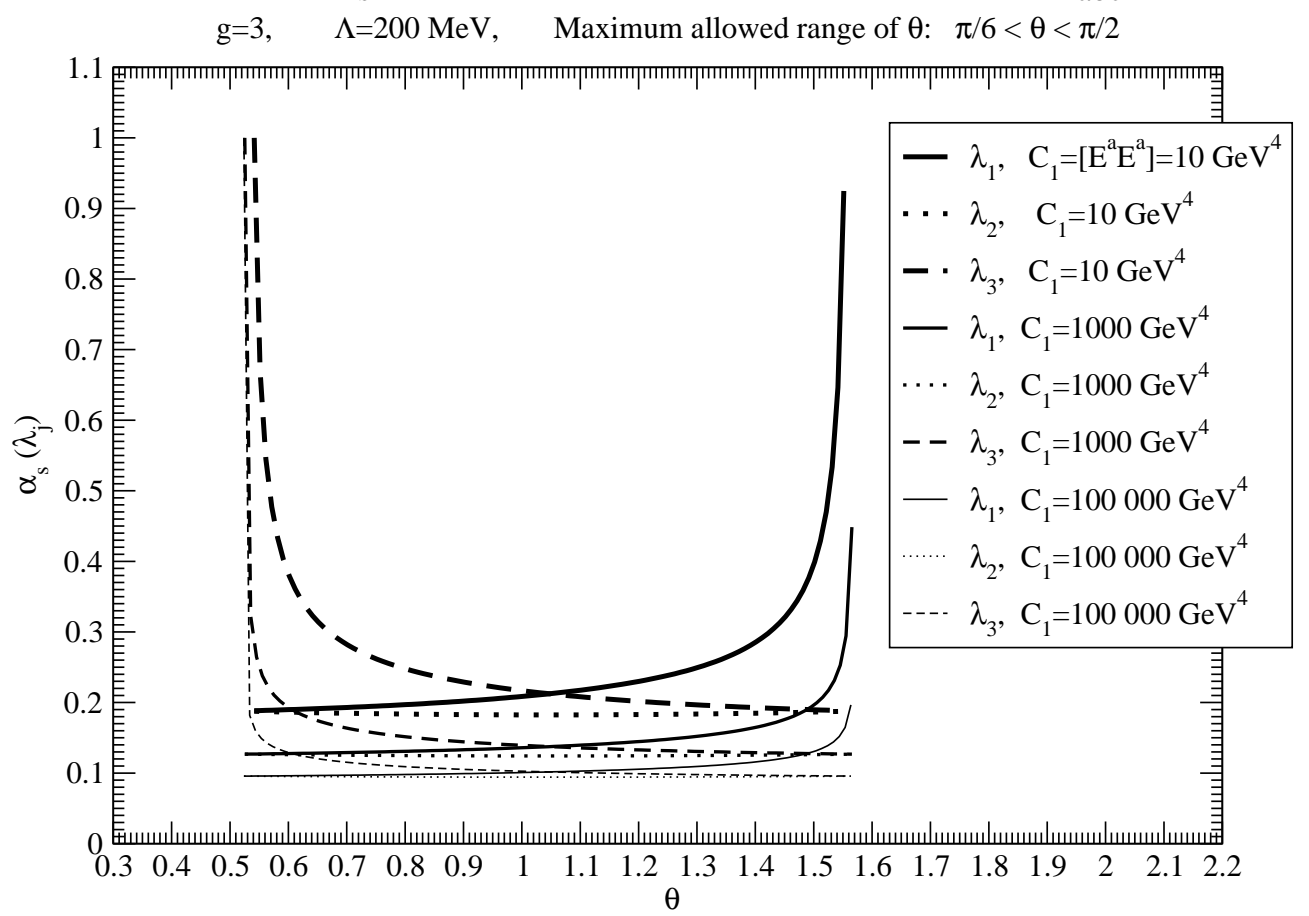

FIG. 2: QCD coupling constant in the presence of $\mathrm{SU}(3)$ chromo-electric field as function $\theta$ for fixed values of first casimir invariant $C_{1}=E^{a} E^{a}$.The $\lambda_{j}$ 's used are from eq. (21). Remember that $\frac{\pi}{6} \leq \theta \leq \frac{\pi}{2}$ is the maximum range when the $\lambda_{j}$ 's are given by eq. (21).

It can be seen from Fig. 1 and Fig. 2 that, for same values of $\theta$, the value of $\alpha_{s}$ decreases as $C_{1}=\left[E^{a} E^{a}\right]$ increases which is consistent with asymptotic freedom.

In Fig. 3 we present the result of $\alpha_{s}\left(\lambda_{j}\right)$ as function of $C_{1}=\left[E_{a} E_{a}\right]$ for fixed values of $\theta$. We have used $g=3$ and $\Lambda=200 \mathrm{MeV}$. The scale $\lambda_{j}$ 's are given by eq. (10). The solid line is the result of $\alpha_{s}\left(\lambda_{1}\right)$ for $\theta=\frac{\pi}{6}$. The dotted line is the result of $\alpha_{s}\left(\lambda_{2}\right)$ for $\theta=\frac{\pi}{3}$ and the dashed line is the result of $\alpha_{s}\left(\lambda_{3}\right)$ for $\theta=\frac{\pi}{2}$. In this figure we have chosen three different 
values of $\theta$ which are within the maximum allowed range $0 \leq \theta \leq \frac{2 \pi}{3}$ as given by eq. (42). It can be seen from Fig. 3 that the $\theta$ dependence is under control in the entire range of $C_{1}=\left[E_{a} E_{a}\right]$.

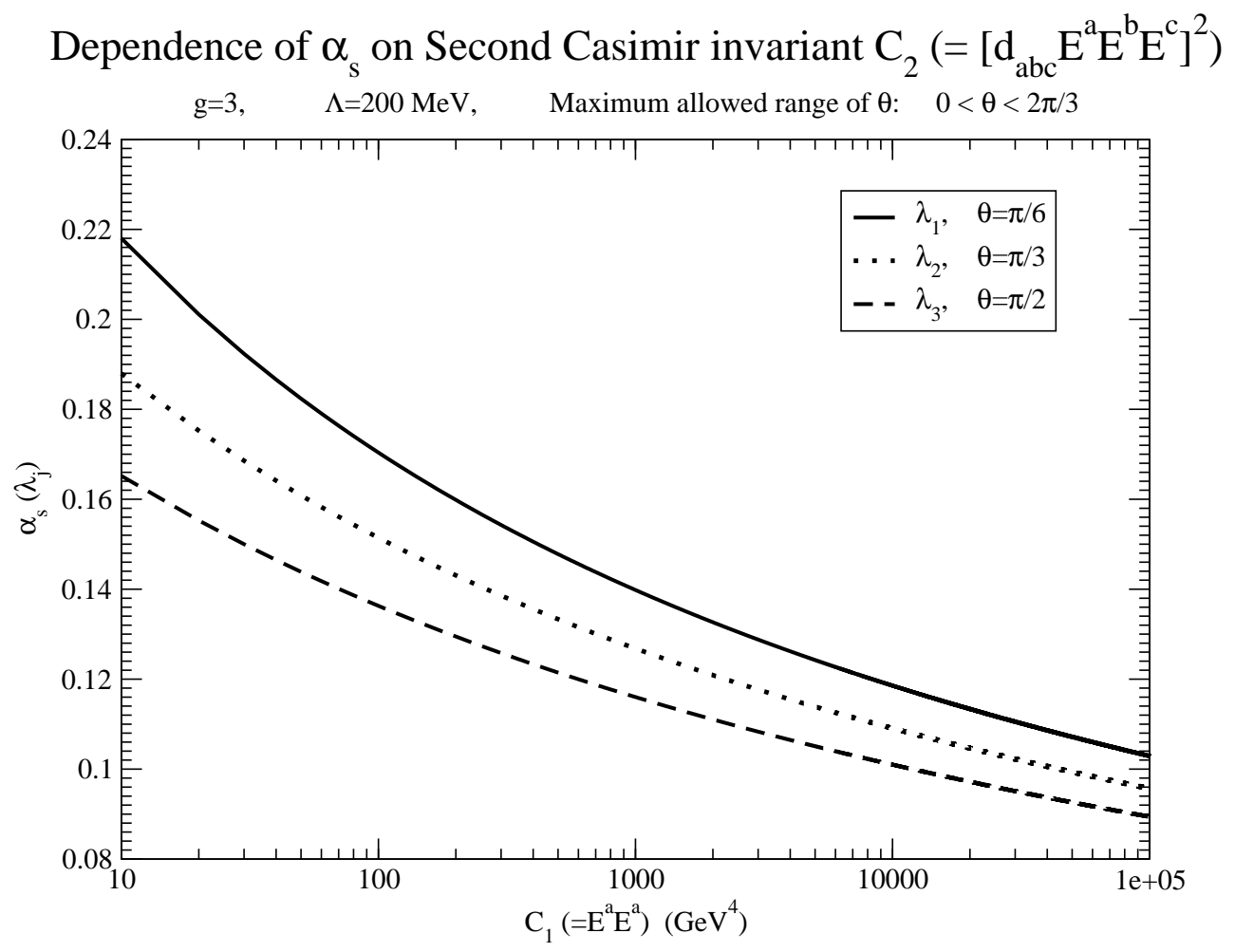

FIG. 3: QCD coupling constant in the presence of $\mathrm{SU}(3)$ chromo-electric field as function of first casimir invariant $C_{1}=\left[E^{a} E^{a}\right]$ for fixed values of $\theta$. The $\lambda_{j}$ 's used are from eq. (10). Remember that $0 \leq \theta \leq \frac{2 \pi}{3}$ is the maximum range when the $\lambda_{j}$ 's are given by eq. (10).

In Fig. 4 we present the result of $\alpha_{s}\left(\lambda_{j}\right)$ as function of $C_{1}=\left[E_{a} E_{a}\right]$ for fixed values of $\theta$. We have used $g=3$ and $\Lambda=200 \mathrm{MeV}$. The scale $\lambda_{j}$ 's are given by eq. (21). The solid line is the result of $\alpha_{s}\left(\lambda_{1}\right)$ for $\theta=\frac{5 \pi}{12}$. The dotted line is the result of $\alpha_{s}\left(\lambda_{3}\right)$ for $\theta=\frac{\pi}{3}$ and the dashed line is the result of $\alpha_{s}\left(\lambda_{2}\right)$ for $\theta=\frac{\pi}{4}$. In this figure we have chosen three different values of $\theta$ which are within the maximum allowed range $\frac{\pi}{6} \leq \theta \leq \frac{\pi}{2}$ as given by eq. (43). It can be seen from Fig. 4 that the $\theta$ dependence is under control in the entire range of $C_{1}=\left[E_{a} E_{a}\right]$.

It can be seen from Fig. 3 and Fig. 4 that the value of $\alpha_{s}$ decreases as $C_{1}=\left[E^{a} E^{a}\right]$ increases which is consistent with asymptotic freedom. 


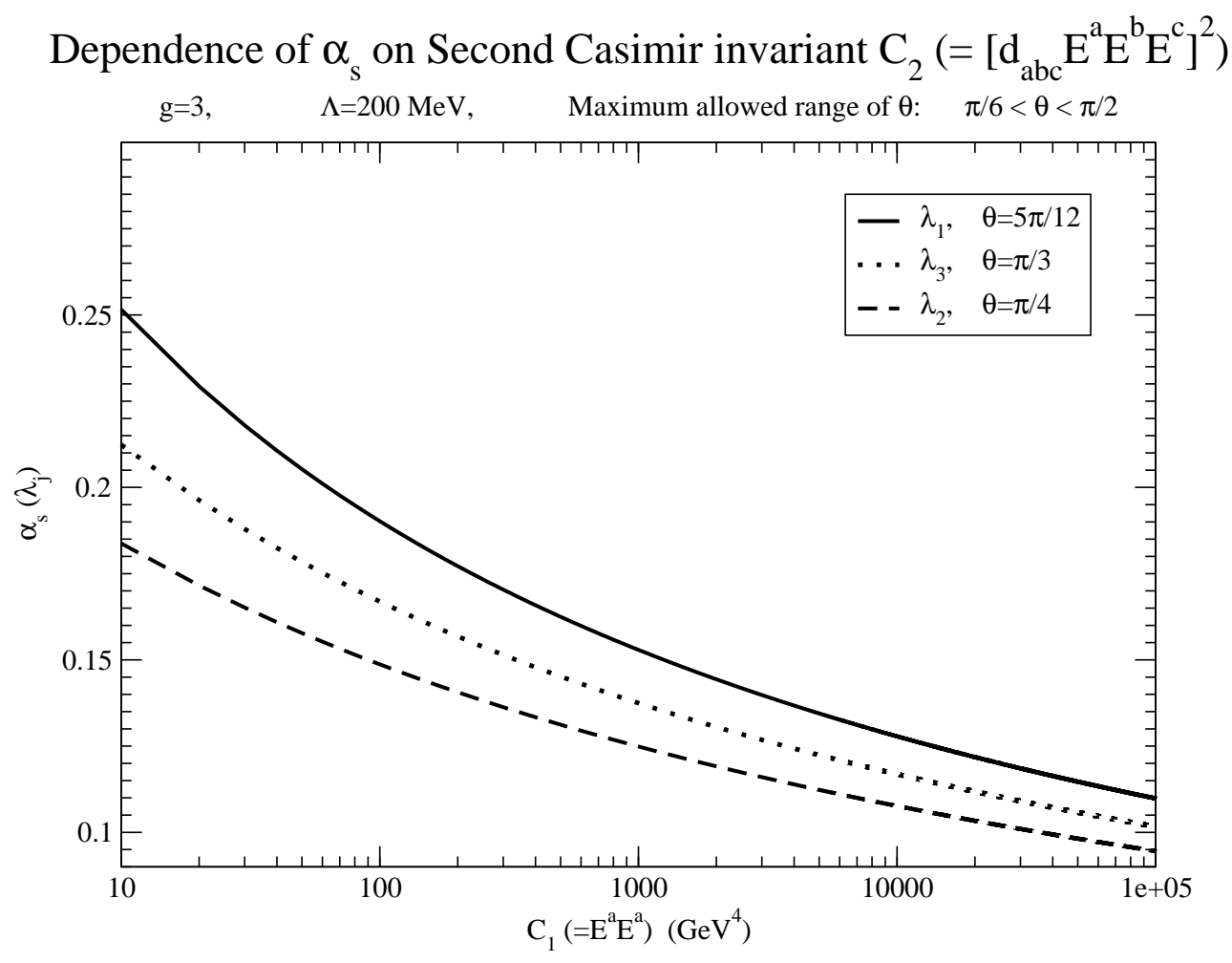

FIG. 4: QCD coupling constant in the presence of SU(3) chromo-electric field as function of first casimir invariant $C_{1}=\left[E^{a} E^{a}\right]$ for fixed values of $\theta$. The $\lambda_{j}$ 's used are from eq. (10). Remember that $\frac{\pi}{6} \leq \theta \leq \frac{\pi}{2}$ is the maximum range when the $\lambda_{j}$ 's are given by eq. (21).

\section{CONCLUSION}

We have solved renormalization group equation in QCD in the presence of $\mathrm{SU}(3)$ constant chromo-electric field $E^{a}$ with arbitrary color index $a=1,2, \ldots 8$. Using background field method in QCD we have obtained the $\beta$ function from one loop effective action of quark and gluon in the presence of chromo-electric field $E^{a}$ in $\mathrm{SU}(3)$. Using these two facts we have determined the exact dependence of the QCD coupling constant on $E^{a}$. We have found that the renormalization scale of the QCD coupling constant $\alpha_{s}$ depends on two independent casimir/gauge invariants $C_{1}=\left[E^{a} E^{a}\right]$ and $C_{2}=\left[d_{a b c} E^{a} E^{b} E^{c}\right]^{2}$ instead of one gauge invariant $C_{1}=\left[E^{a} E^{a}\right]$. These coupling constant may play an important role in the study of production and equilibration of quark-gluon plasma from classical color field at RHIC and LHC. This coupling constant may also play an important role to study low $p_{T}$ hadron production at collider experiments using string breaking mechanism in the color flux-tube model. 


\section{Acknowledgments}

This work was supported in part by Department of Energy under contracts DE-FG0291ER40664, DE-FG02-04ER41319 and DE-FG02-04ER41298.

[1] G. C. Nayak and P. van Nieuwenhuizen, Phys. Rev. D71 (2005) 125001; F. Cooper and G. C. Nayak, Phys. Rev. D73 (2006) 065005; G. C. Nayak, Phys. Rev. D72 (2005) 125010.

[2] J. Schwinger, Phys. Rev. 82 (1951) 664.

[3] W. Heisenberg and H. Euler, Z. Physik 98, 714 (1936).

[4] A. Casher, H. Neuberger and S. Nussinov, Phys. Rev. D 20, 179 (1979).

[5] B. Andersson, the Lund model, Cambridge University Press, Cambridge, 1998); PYTHIA: http://www.thep.lu.se/(tilde)torbjorn/pythia.html

[6] F. Cooper, E. Mottola and G. C. Nayak, Phys. Lett. B 555, 181 (2003); G. C. Nayak et al., Nucl. Phys. A687, 457 (2001).

[7] L. McLerran and R. Venugopalan, Phys. Rev. D49 (1994) 2233; Phys. Rev. D49 (1994) 3352;

E. Iancuu, A. Leonidov and L. McLerran, Phys. Lett. B510 (2001) 133; E. Iancuu, A. Leonidov and L. McLerran, Nucl. Phys. A692 (2001) 583; J. Jalilian-Marian, A. Kovner, L. Mcerran and H. Weigert, Phys. Rev. D55 (1997) 5414.

[8] G. 't Hooft, Nucl. Phys. B62 (1973) 444.

[9] L. F. Abbott, Nucl. Phys. B185 (1981) 189.

[10] S. Coleman and E. Weinberg, Phys. Rev. D 7 (1973) 1888.

[11] T. Muta, Foundations of Quantum Chromodynamics, (World scientific lecture notes in physicsvol. 5).

[12] For a list of non-zero values of $d_{a b c}$, see for example, Particle Data Group, http://pdg.lbl.gov/. 